\title{
Development of a valid measurement instrument to understand self-regulatory driving practices among older drivers in Malaysia
}

\begin{abstract}
Self-regulatory driving is a term used to describe a strategy used by older drivers to preserve mobility and safety, through the adjustment of driving behaviors to match declining physical functions. It can be regarded as a way to prolong driving, or as a process leading to the cessation of driving. Previous studies have striven to explore and understand how older drivers self-regulate their driving. This paper aims to provide an overview of the relevant theories, to explicate the factors that contribute to the adoption of self-regulated driving and the scales used to measure self-regulatory behaviors. This paper also reports on the development and psychometric testing of a Self-Regulatory Driving Practices (SRDP) scale in the Malaysian context. Based on the reviewed theories, adoption of self-regulatory driving practices is a process and involves cognitive thinking that reflects a set of actions. Existing instruments to measure self-regulatory driving practices have been developed and used to identify the behavioral components of self-regulation. Based on literature reviews and a thematic analysis from focus group discussions, a SRDP scale was developed, accommodating the Malaysian context. There were 498 surveys completed by older drivers for further psychometric testing purposes. Results revealed that the final 12-item SRDP scale $(\alpha=0.81)$ consists of four subscales that are planning, avoidance, reduction and alternatives. Suggestions for future research are also recommended.
\end{abstract}

Keyword: Older drivers; Self-regulatory driving; Theory; Measure; Scale development 